\title{
GMR
}

\section{Association between MYC rs9642880[T] allele and bladder cancer risk: a meta-analysis}

\author{
Y. Zhao ${ }^{1 *}$, J.G. Qi ${ }^{1 *}$, N. Yang ${ }^{1}$, Y.L. Lin ${ }^{1}$, J. Liang ${ }^{1}$ and X. Zhu ${ }^{2}$ \\ 1'Department of Urology, Xuzhou Cancer Hospital, \\ Affiliated Hospital of Jiangsu University, Jiangsu, China \\ ${ }^{2}$ Department of General Surgery, \\ The East District of Suzhou Municipal Hospital, Suzhou, China \\ ${ }^{*}$ These authors contributed equally to this study. \\ Corresponding author: X. Zhu \\ E-mail: 601127797@qq.com
}

Genet. Mol. Res. 14 (4): 14745-14751 (2015)

Received May 6, 2015

Accepted July 13, 2015

Published November 18, 2015

DOI http://dx.doi.org/10.4238/2015.November.18.39

\begin{abstract}
A single nucleotide polymorphism of MYC rs9642880 (G>T) at the $8 \mathrm{q} 24.1$ locus is thought to be associated with bladder cancer risk based on the results of genome-wide association studies, but the results remain inconclusive. To assess the association between rs9642880[T] allele and bladder cancer risk, we performed this meta-analysis including 18 case-control studies and involving 23,084 cases and 97,164 controls. Electronic searches for publications were conducted to determine the association between this variant and prostate cancer in several databases. The last search update was August 4, 2014. We used odds ratios and $95 \% \mathrm{Cls}$ to evaluate the strength of the associations. The overall results suggested that the rs9642880[T] allele was associated with bladder cancer susceptibility $(T$ vs $\mathrm{G}$, odds ratio $=1.18,95 \% \mathrm{Cl}=1.14-1.22)$. In subgroup analysis by ethnicity and source of controls, the risk remained significant. The present meta-analysis suggests that the MYC rs9642880[T] allele is significantly associated with bladder cancer risk.
\end{abstract}

Key words: Bladder cancer; Meta-analysis; MYC; rs9642880[T] allele 


\section{INTRODUCTION}

Urinary bladder cancer is the 9th most common cancer (Golka et al., 2011). There were approximately 386,300 new cases and 150,200 deaths from bladder cancer in 2008 worldwide, with most cases occurring in males (Jemal et al., 2011). Bladder cancer is a complex and heterogeneous disease caused by interactions between both environmental and genetic factors. The strongest known environmental risk factors include occupational exposure to bladder carcinogens, cigarette smoking, and male gender. Numerous inherited genetic variants have been reported to be associated with bladder cancer risk, but few of these candidate-gene associations have been consistently replicated (Chang et al., 2012), indicating that the precise molecular mechanisms of bladder cancer are unclear.

Several studies have identified numerous single-nucleotide polymorphisms on chromosome 8q24 that are associated with the risk of breast, prostate, and colorectum cancers (Easton et al., 2007; Gudmundsson et al., 2007; Zanke et al., 2007), indicating that genetic variation in this region may play an important role in the etiology of numerous cancers. Recently, genomewide association studies have reported that the 8q24 single-nucleotide polymorphism rs9642880 is associated with risk of urinary bladder cancer (Golka et al., 2009; Wang et al., 2009; Schwender et al., 2012; Yates et al., 2013; Wang et al., 2014a). The rs9642880 (G>T) variant is located in the linkage disequilibrium block and only $30 \mathrm{~kb}$ upstream of MYC (Kiemeney et al., 2008; Golka et al., 2009). MYC is a multifaceted protein that regulates cell proliferation, differentiation, and apoptosis, and plays an important role in various cancers (DePinho et al., 1991). In addition, MYC, which is activated in $70 \%$ of all human cancers, is upregulated in bladder cancer and is thought to promote tumor progression (Sauter et al., 1995; Nilsson and Cleveland, 2003). In recent years, the single nucleotide polymorphism MYC is rs9642880 (G>T) has been extensively examined.

Several studies have focused on the association between the minor [T] allele of rs 9642880 and bladder cancer susceptibility (Golka et al., 2009; Wu et al., 2009; Schwender et al., 2012; Yates et al., 2013; Wang et al., 2009, 2014a,b). Many of these studies showed that the rs9642880[T] allele on 8q24 had the strongest association with the risk of bladder cancer. However, Yates et al. (2013) found that the [T] allele of rs9642880 was not associated with adverse pathological variables, indicating that the results are inconclusive. Thus, we performed a meta-analysis to more precisely estimate this association between the MYC rs9642880 polymorphism and bladder cancer risk.

\section{MATERIAL AND METHODS}

\section{Identification and eligibility of relevant studies}

We searched the electronic database PubMed for all relevant articles (the last search update was August 4, 2014) using the following search terms: "MYC" or "rs9642880", "genetic variant" or "polymorphism", "bladder cancer" or "carcinoma of bladder". The search was limited to English-language articles. Additional studies were identified by a manual search of the references of original studies. All relevant publications were reviewed. When more than one study of the same population was included in several publications, only the most recent or complete study was used in this meta-analysis. As a result, 8 eligible case-control studies were included in our meta-analysis. 


\section{Data extraction}

Two investigators independently extracted information from all eligible publications. Discrepancies were adjudicated by a third reviewer until consensus was achieved on every item. For each eligible study, the following information was recorded: first author's name, year of publication, country of origin, ethnicity, source of control groups (mixed-, population-, or hospital-based controls), genotyping method, and number of cases and controls. For studies including subjects of different ethnic groups, data were extracted separately for each ethnic group whenever possible. Different ethnicities were categorized as European and Asian. MYC locus polymorphism studies provided information for the allele of $\mathrm{G}$ or $\mathrm{T}$ of rs9642880.

\section{Statistical analysis}

The strength of the association between the MYC rs9642880 polymorphism and bladder cancer risk was measured by odds ratios (ORs) with $95 \% \mathrm{Cls}$. To examine heterogeneity across the studies, a statistical test for heterogeneity was performed based on the $Q$ statistic (Handoll, 2006). If the $P$ value was $>0.05$, the $Q$ test indicated a lack of heterogeneity among studies, and the summary OR estimate of each study was calculated using the fixed-effect model (the Mantel-Haenszel method) (Mantel and Haenszel, 1959). Otherwise, the randomeffect model (the DerSimonian and Laird method) was used (DerSimonian and Laird, 1986). Stratified analyses were also performed by ethnicity and source of controls. Sensitivity analyses were performed to assess the stability of the results. A single study in the meta-analysis was deleted individually to determine the influence of an individual data set on the pooled OR. In addition, both funnel plot and the Egger test were used to assess publication bias $(P<0.10$ was considered statistically significant) (Egger et al., 1997). All statistical analyses were performed in the Statistical Analysis the System software (version 11.0; StataCorp LP, College Station, TX, USA), using 2-sided $P$ values.

\section{RESULTS}

\section{Characteristics of studies}

In total, 18 eligible case-control studies involving 23,084 cases and 97,164 controls were included in this meta-analysis (Kiemeney et al., 2008; Golka et al., 2009; Wu et al., 2009; Schwender et al., 2012; Yates et al., 2013; Wang et al., 2009, 2014a,b). The characteristics of studies selected are summarized in Table 1. These eligible publications included studies from France, Germany, Iceland, Netherland, UK, Italy, Belgium, Sweden, Spain and other Europeans, US, and China populations. All studies were case-control studies. There were 4 studies of Asians and 14 studies of Europeans. Bladder cancers were confirmed histologically or pathologically in most studies. The TaqMan assay was performed in 6 studies. Moreover, controls were age- and gender-matched in most studies, 5 of which were population-based, 10 were hospital-based, and 3 were mixed. The distribution of the rs9642880[T] allele among bladder cancer cases and controls of all studies are shown in Table 1. 


\section{Quantitative synthesis}

The main results of this meta-analysis and the heterogeneity tests are shown in Table 2. Significant associations were observed between the rs9642880[T] allele and risk of bladder cancer in the overall population ( $T$ vs $\mathrm{G}, \mathrm{OR}=1.18,95 \% \mathrm{Cl}=1.14-1.22, \mathrm{P}_{\text {heterogeneity }}=0.797$ ). Furthermore, in the stratified analysis, there were significantly increased risks among studies with populationbased controls $\left(\mathrm{OR}=1.16,95 \% \mathrm{Cl}=1.09-1.24, \mathrm{P}_{\text {heterogeneity }}=0.859\right)$ and hospital-based controls $\left(\mathrm{OR}=1.23,95 \% \mathrm{Cl}=1.16-1.32, \mathrm{P}_{\text {heterogeneity }}=0.643\right)($ Table 2$)$. In addition, we analyzed subgroups by ethnicity (Europeans and Asians). A significant association was also detected in bladder cancer among Europeans $\left(\mathrm{OR}=1.18,95 \% \mathrm{Cl}=1.14-1.23, \mathrm{P}_{\text {heterogeneity }}=0.755\right)$ and Asians $(\mathrm{OR}=1.19$, $\left.95 \% \mathrm{Cl}=1.10-1.28, \mathrm{P}_{\text {heterogeneity }}=0.425\right)($ Table 2$)$.

\begin{tabular}{|c|c|c|c|c|c|c|c|c|c|c|}
\hline \multirow[t]{2}{*}{ First author (year) } & \multirow[t]{2}{*}{ Country } & \multirow[t]{2}{*}{ Ethnicity } & \multirow[t]{2}{*}{ Source of controls } & \multirow[t]{2}{*}{ Genotyping method } & \multicolumn{3}{|c|}{ Cases } & \multicolumn{3}{|c|}{ Controls } \\
\hline & & & & & Total & G & $\mathrm{T}$ & Total & G & $\mathrm{T}$ \\
\hline Wang et al. (2014a) & China & Asian & Population & TaqMan & 2420 & 1636 & 784 & 2016 & 1417 & 599 \\
\hline Holger et al. (2012) & Multi-country & European & Mixed & TaqMan & 3168 & 1549 & 1619 & 3476 & 1848 & 1628 \\
\hline David et al. (2013) & France & European & Hospital & TaqMan & 462 & 242 & 220 & 522 & 292 & 230 \\
\hline \multirow[t]{2}{*}{ Klaus et al. (2009) } & Germany & European & Hospital & TaqMan & 424 & 187 & 237 & 388 & 201 & 187 \\
\hline & Germany & European & Population & TaqMan & 606 & 299 & 307 & 1398 & 720 & 678 \\
\hline \multirow[t]{2}{*}{ Meilin et al. (2009) } & China & Asian & Hospital & PCR-RFLP & 460 & 276 & 184 & 510 & 349 & 161 \\
\hline & China & Asian & Hospital & PCR-RFLP & 370 & 225 & 145 & 420 & 289 & 131 \\
\hline \multirow[t]{9}{*}{ Lambertus et al. (2008) } & Iceland & European & Mixed & Whole-Genome & 1046 & 492 & 554 & 64916 & 33756 & 31160 \\
\hline & Netherland & European & Population & Whole-Genome & 2538 & 1193 & 1345 & 3648 & 1897 & 1751 \\
\hline & UK & European & Hospital & Single-Track Assay & 1390 & 709 & 681 & 1014 & 558 & 456 \\
\hline & Italy & European & Hospital & Single-Track Assay & 646 & 323 & 323 & 762 & 450 & 312 \\
\hline & Italy & European & Hospital & Single-Track Assay & 348 & 195 & 153 & 366 & 216 & 150 \\
\hline & Belgium & European & Population & Single-Track Assay & 390 & 199 & 191 & 744 & 409 & 335 \\
\hline & Multi-country & European & Hospital & Single-Track Assay & 372 & 179 & 193 & 1030 & 577 & 453 \\
\hline & Sweden & European & Population & Single-Track Assay & 638 & 313 & 325 & 1818 & 945 & 873 \\
\hline & Spain & European & Hospital & Single-Track Assay & 342 & 178 & 164 & 1672 & 920 & 752 \\
\hline Wu et al. (2009) & USA & European & Mixed & Mixed & 5364 & 2746 & 2618 & 9656 & 5243 & 4413 \\
\hline Wang et al. (2014b) & China & Asian & Hospital & TaqMan & 2100 & 920 & 1180 & 2808 & 1344 & 1464 \\
\hline
\end{tabular}

\begin{tabular}{|c|c|c|c|c|}
\hline $\begin{array}{l}\text { Variables } \\
\text { T vs G }\end{array}$ & $N_{\mathrm{A}}$ & $\begin{array}{l}\text { Sample size } \\
\text { (case/control) }\end{array}$ & OR $(95 \% \mathrm{Cl})$ & $P$ value $^{b}$ \\
\hline Total & 18 & $23,084 / 97,164$ & $1.18^{*}(1.14-1.22)$ & 0.797 \\
\hline \multicolumn{5}{|l|}{ Ethnicities } \\
\hline European & 14 & $17,734 / 91,410$ & $1.18^{*}(1.14-1.23)$ & 0.755 \\
\hline Asian & 4 & $5,350 / 2,754$ & $1.19^{*}(1.10-1.28)$ & 0.425 \\
\hline \multicolumn{5}{|l|}{ Source of controls } \\
\hline Population-based & 5 & $6,592 / 9,624$ & $1.16^{\star}(1.09-1.24)$ & 0.859 \\
\hline Hospital-based & 10 & $6,914 / 9,492$ & $1.23^{\star}(1.16-1.32)$ & 0.643 \\
\hline
\end{tabular}

$\mathrm{Cl}=$ confidence interval; $\mathrm{OR}=$ odds ratio. ${ }^{*} \mathrm{P}<0.05$. aNumber of comparisons. ${ }^{\mathrm{b}} \mathrm{P}$ value of $\mathrm{Q}$-test for heterogeneity test.

\section{Test of heterogeneity and sensitivity analyses}

No significant heterogeneity between studies was detected in overall comparisons (Table 2). Moreover, a single study involved in the meta-analysis was deleted to reflect the influence of 
the individual data set on the pooled ORs, and the corresponding pooled ORs were not materially altered (data not shown), suggesting that the results of this meta-analysis were stable.

\section{Publication bias}

Begg's funnel plot and the Egger test were used to assess publication bias. The shape of the funnel plots revealed no evidence of obvious asymmetry in the overall meta-analysis (Figure 1 shows the funnel plot of overall $T$ vs G). Next, to provide statistical evidence of funnel plot symmetry, the Egger test was performed. The results still did not present any obvious evidence of publication bias $(P>0.05)$.

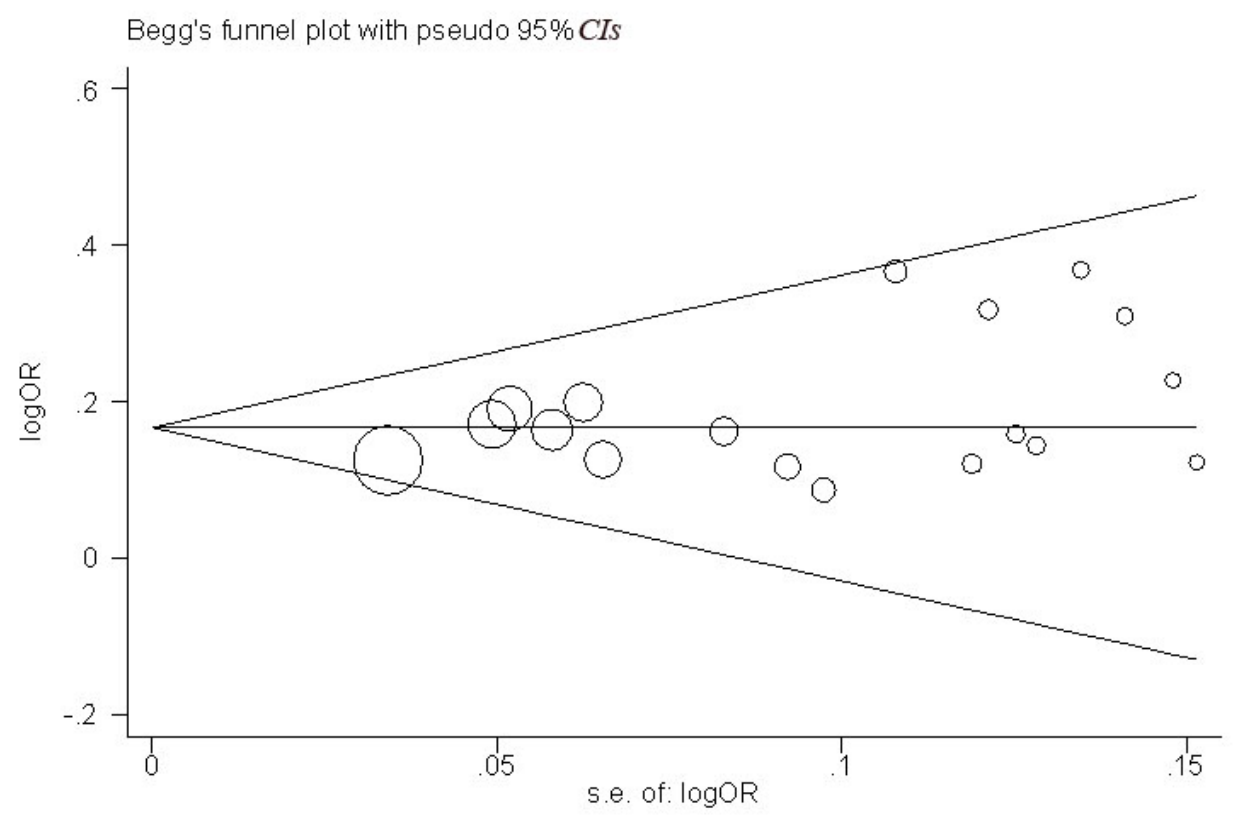

Figure 1. Begg's funnel plot analysis with pseudo $95 \%$ confidence limits to detect publication bias (T vs G). Each point represents a separate study for the indicated association. Log[or], natural logarithm of odds ratio. Horizontal line, mean effect size.

\section{DISCUSSION}

The present meta-analysis, including 23,084 cases and 97,164 controls from 18 casecontrol studies, explored the association between the MYC rs9642880[T] allele and bladder cancer risk. This is the most comprehensive meta-analysis that has investigated whether the MYC rs9642880[T] allele at the 8q24.1 locus is associated with bladder cancer risk. Our results showed that the rs9642880[T] allele was associated with an increased risk of bladder cancer. Given the important roles of MYC in regulating cell proliferation, it is biologically plausible that genetic variations in MYC rs9642880 (G>T) may modulate the risk of bladder cancer.

Cancer is a multifactorial disease that results from complex interactions between many inherited and environmental factors (Pharoah et al., 2004). In contrast to familial cancer syndromes, 
these factors generally increase the risk of sporadic forms of cancer that tend to be common in the population. Genetic variations in DNA repair genes can adjust the DNA repair capacity and alter cancer risk. Known genes account for a small proportion of cancer risk, and approximately 150 human DNA repair genes have been identified (Wood et al., 2005). However, the actual number of human DNA repair genes is likely to be higher, and many genes with modest effects remain to be identified.

MYC, also known as c-MYC, is a nuclear phosphoprotein transcription factor that plays an important role in cell apoptosis and proliferation. In addition, MYC regulates approximately $10 \%$ of human genes (Fernandez et al., 2003) and have the ability to promote cellular proliferation and block cellular differentiation. Dysregulation of MYC has been detected in a wide variety of human tumors, such as prostate cancer, breast cancer, and osteogenic sarcoma (Anso et al., 2013; Zhang et al., 2014; Terunuma et al., 2014). For the past few years, MYC genetic variations have received widespread attention, particularly the single nucleotide polymorphism MYC rs9642880 (G>T). Recently, several studies have investigated the association between the MYC rs9642880[T] allele and bladder cancer susceptibility in different countries (Kiemeney et al., 2008; Golka et al., 2009; Wu et al., 2009; Schwender et al., 2012; Yates et al., 2013; Wang et al., 2009, 2014a,b). However, the results are inconclusive, partially because of the possible small effect of the polymorphism on bladder cancer risk and the relatively small sample sizes in each of the published studies. Therefore, to more precisely estimate the association, a meta-analysis including 23,084 cases and 97,164 controls from 18 case-control studies was performed. Our results suggest that the MYC rs9642880[T] variant genotype is significantly associated with the risk of bladder cancer in the overall studied populations.

In stratified analysis by ethnicity, we detected a significant association in both Europeans and Asians, suggesting that ethnic differences in genetic backgrounds and the environmental/ lifestyle context do not affect the association between the MYC rs9642880[T] allele associated and bladder cancer risk. The same result was also found in the stratified analysis in population-based and hospital-based controls, indicating that including a different source of controls did not influence the association.

There were several limitations to our analysis. First, the overall outcomes were based on individual unadjusted ORs, while a more precise evaluation should be adjusted by other potentially suspected factors (i.e., smoking, age, and environmental factors). Second, because the eligible studies included in the meta-analysis were only those that have been published, publication bias may have occurred, although statistical analysis of the data did not show this effect. Despite these limitations, the meta-analysis also had some advantages. First, the results may be unbiased as no publication biases were detected. Second, the number of total cases and controls was substantial, which significantly increased the statistical power of this study.

In conclusion, based on our larger sample size, the results of this meta-analysis suggests that rs9642880[T] is a risk factor for bladder cancer. The MYC rs9642880[T] allele may be an independent risk factor for bladder cancer. Nevertheless, additional larger studies, particularly studies stratified for gene-environmental interactions, should be carried out to confirm our findings.

\section{Conflicts of interest}

The authors declare no conflict of interest. 


\section{ACKNOWLEDGMENTS}

This study was not supported by any financial. The authors were fully responsible for all content and editorial decisions.

\section{REFERENCES}

Anso E, Mullen AR, Felsher DW, Matés JM, et al. (2013). Metabolic changes in cancer cells upon suppression of MYC. Cancer Metab. 1: 7.

Chang DW, Gu J and Wu X (2012). Germline prognostic markers for urinary bladder cancer: obstacles and opportunities. Urol. Oncol. 30: 524-532.

DePinho RA, Schreiber-Agus N and Alt FW (1991). myc family oncogenes in the development of normal and neoplastic cells. Adv. Cancer Res. 57: 1-46.

DerSimonian R and Laird N (1986). Meta-analysis in clinical trials. Control Clin. Trials 7: 177-188.

Easton DF, Pooley KA, Dunning AM, Pharoah PD, et al. (2007). Genome-wide association study identifies novel breast cancer susceptibility loci. Nature 447: 1087-1093.

Egger M, Davey Smith G, Schneider M and Minder C (1997). Bias in meta-analysis detected by a simple, graphical test. BMJ 315: 629-634.

Fernandez PC, Frank SR, Wang L, Schroeder M, et al. (2003). Genomic targets of the human c-Myc protein. Genes Dev. 17: 1115-1129.

Golka K, Hermes M, Selinski S, Blaszkewicz M, et al. (2009). Susceptibility to urinary bladder cancer: relevance of rs9642880[T], GSTM1 0/0 and occupational exposure. Pharmacogenet. Genomics 19: 903-906.

Golka K, Selinski S, Lehmann ML, Blaszkewicz M, et al. (2011). Genetic variants in urinary bladder cancer: collective power of the "wimp SNPs". Arch. Toxicol. 85: 539-554.

Gudmundsson J, Sulem P, Manolescu A, Amundadottir LT, et al. (2007). Genome-wide association study identifies a second prostate cancer susceptibility variant at 8q24. Nat. Genet. 39: 631-637.

Handoll HH (2006). Systematic reviews on rehabilitation interventions. Arch. Phys. Med. Rehabil. 87: 875.

Jemal A, Bray F, Center MM, Ferlay J, et al. (2011). Global cancer statistics. CA Cancer J. Clin. 61: 69-90.

Kiemeney LA, Thorlacius S, Sulem P, Geller F, et al. (2008). Sequence variant on 8q24 confers susceptibility to urinary bladder cancer. Nat. Genet. 40: 1307-1312.

Mantel $\mathrm{N}$ and Haenszel W (1959). Statistical aspects of the analysis of data from retrospective studies of disease. J. Natl. Cancer Inst. 22: 719-748.

Nilsson JA and Cleveland JL (2003). Myc pathways provoking cell suicide and cancer. Oncogene 22: 9007-9021.

Pharoah PD, Dunning AM, Ponder BA and Easton DF (2004). Association studies for finding cancer-susceptibility genetic variants. Nat. Rev. Cancer 4: 850-860.

Sauter G, Carroll P, Moch H, Kallioniemi A, et al. (1995). c-myc copy number gains in bladder cancer detected by fluorescence in situ hybridization. Am. J. Pathol. 146: 1131-1139.

Schwender H, Selinski S, Blaszkewicz M, Marchan R, et al. (2012). Distinct SNP combinations confer susceptibility to urinary bladder cancer in smokers and non-smokers. PLoS One 7: e51880.

Terunuma A, Putluri N, Mishra P, Mathé EA, et al. (2014). MYC-driven accumulation of 2-hydroxyglutarate is associated with breast cancer prognosis. J. Clin. Invest. 124: 398-412.

Wang M, Wang M, Zhang W, Yuan L, et al. (2009). Common genetic variants on 8q24 contribute to susceptibility to bladder cancer in a Chinese population. Carcinogenesis 30: 991-996.

Wang M, Chu H, Lv Q, Wang L, Yuan L, et al. (2014a). Cumulative effect of genome-wide association study-identified genetic variants for bladder cancer. Int. J. Cancer 135: 2653-2660.

Wang P, Ye D, Guo J, Liu F, et al. (2014b). Genetic score of multiple risk-associated single nucleotide polymorphisms is a marker for genetic susceptibility to bladder cancer. Genes Chromosomes Cancer 53: 98-105.

Wood RD, Mitchell M and Lindahl T (2005). Human DNA repair genes, 2005. Mutat. Res. 577: 275-283.

Wu X, Ye Y, Kiemeney LA, Sulem P, et al. (2009). Genetic variation in the prostate stem cell antigen gene PSCA confers susceptibility to urinary bladder cancer. Nat. Genet. 41: 991-995.

Yates DR, Rouprêt M, Drouin SJ, Audouin M, et al. (2013). Genetic polymorphisms on 8q24.1 and 4p16.3 are not linked with urothelial carcinoma of the bladder in contrast to their association with aggressive upper urinary tract tumours. World J. Urol. 31: 53-59.

Zanke BW, Greenwood CM, Rangrej J, Kustra R, et al. (2007). Genome-wide association scan identifies a colorectal cancer susceptibility locus on chromosome 8q24. Nat. Genet. 39: 989-994.

Zhang C, Zhang S, Zhang Z, et al. (2014). MYC-driven accumulation of 2-hydroxyglutarate is associated with breast cancer prognosis. J. Clin. Invest. 124: 398-412. 